E.L.U.A., 11, 1996-97, pp: $385-409$

\title{
ESQUEMAS ORACIONALES ERGATIVOS REFLEXIVOS ${ }^{1}$
}

\author{
Agustín Vera LuJán \\ (Universidad de Murcia)
}

\begin{abstract}
RESUMEN
This work poses an explanation of ergative-reflexive sentences, according to which the peculiarities and, particularly, the changes involved in the valencies patterns of the verbs to be used in these sentences are the result of the partial matching of the reflexive prototype SE. This function of SE seems to be fully coherent with other uses of SE, either prototypical or non-prototypical.
\end{abstract}

El objetivo de este trabajo lo constituye el estudio de una serie de esquemas oracionales como los que se manifiestan en enunciados del tipo de (1)-(6) ${ }^{2}$, denominables, siguiendo a Burzio (1986), ergativos.

(1)....bisagras y aldabas se torcían de calor (CAS, p. 28)

(2)... el pueblo se alegraba con los acordes progresivos de una misma pieza (CAS, p. 40)

(3) Al principio nadie se alarmó (CAS, p. 46)

(4) Las mieses también se han agostado por falta de lluvias oportunas (CONF, p. 84)

(5)... hubo un pequeño viejo, mi bisabuelo, que se afligió profundamente (CONF, p. 77)

(6) El tren se ha puesto otra vez en marcha, y se aleja con un sordo fragor (PUE, p. 72 ) CAM.

El presente trabajo ha sido realizado, en parte, gracias a una ayuda de la Fundación Cultural

2 Los ejemplos citados en este trabajo provienen de G.García Márquez, Cien años de soledad, Buenos Aires, Editorial Sudamericana, 1968, en adelante (CAS), R. Gómez de la Serna, Flor de Greguerías, Buenos Aires, Losada, 1956 (FLOR), y de las siguientes obras de Azorín: Confesiones de un pequeño filósofo, Madrid, Librería de Fernando $\mathrm{Fe}$ 1904, (CONF), y Los pueblos. La andalucía trágica y otros artículos, edic. de José María Valverde, Madrid, Clásicos Castalia, 1974, (PUE). 
Semejantes enunciados son manifestación de una serie de estructuras oracionales cuyo funcionamiento ha sido esclarecido de manera satisfactoria en lo que se refiere a sus aspectos descriptivos, pero respecto de las cuales falta aún, en nuestra opinión, una hipótesis explicativa razonablemente adecuada sobre el por qué de la presencia en tales oraciones del átono reflexivo de tercera persona $\mathrm{SE}$, de la relación de dicha forma con el resto de formas reflexivas (especialmente con la prototípicamente reflexiva) y de la naturaleza de los mecanismos que vinculan a dicho elemento con los procesos de transclasificación que afectarían a la categorización paradigmática de los verbos presentes en las oraciones en cuestión.

Enunciados como los de (1) a (6), que denominaremos en adelante ergativos reflexivos, presentan, en efecto, particularidades descriptivas que les diferencian del resto de esquemas reflexivos.

En los enunciados prototípicamente reflexivos, como (7) u (8), el átono reflexivo tiene un papel funcional oracional relacionado con el de complemento directo - véase la equivalencia (7)/(7i) - o el de complemento indirecto - (8)/ (8i) - : como indicábamos en Vera (1990), la forma átona de tercera persona constituye en tales casos un monema verbal objetivo gracias al cual es posible marcar la concordancia entre el verbo y el sintagma en función de complemento directo o indirecto, siempre nucleado por las formas tónicas correspondientes que, aunque no necesariamente hayan de manifestarse, se encuentran funcionalmente operativas en sus esquemas oracionales, estructuralmente equivalentes, por tanto, a (7ii) y (8ii).

(7) El señor Lletget, presunto buen mozo que SE afeita de siete en siete días... (PUE, 53)

(7i) El señor Lletget, presunto buen mozo que LO afeita de siete en siete días

(7ii) El señor Lletget, presunto buen mozo que se afeita (A Sí MISMO) de siete en siete dias...

(8) Úrsula SE ponía antes de acostarse un pantalón rudimentario (CAS, 25)

(8i) Úrsula LE ponía antes de acostarse un pantalón rudimentario

(8ii) Úrsula se ponía (A SÍ MISMA) antes de acostarse un pantalón rudimentario.

El SE presente en las estructuras ergativas reflexivas carece de la funcionalidad que caracteriza a esta forma átona en los esquemas prototípicamente reflexivos: no constituye marca significante alguna de las funciones oracionales de complemento directo o indirecto (como mostraría la no equivalencia de estas oraciones con las correspondientes de una serie en las que la forma pronominal cumpliera dicha función y fuera, por tanto, conmutable por formas átonas característicamente asociadas a tales funciones como LO(S)/LA(S) y LE(S)), y, por consiguiente, tampoco de complementos directos o indirectos correferenciales con el sujeto — véanse, a título de ejemplo, (1i-ii) y (6i-ii)-: 
(Ii)... * bisagras y aldabas LO torcían de calor

(1ii).... *bisagras y aldabas se torcían A Sí MISMAS de calor

(6i) *El tren LO ha puesto otra vez en marcha

(6ii) *El tren se ha puesto A SÍ MISMO otra vez en marcha

Construidas sobre la base de verbos paradigmáticamente transitivos (torcer, alegrar, alarmar, agostar, afligir...), las estructuras ergativas reflexivas contrastan, sin embargo, con las reflexivas prototípicas como (7) u (8), necesariamente transitivas. El átono $\mathrm{SE}$ de las construcciones ergativas reflexivas está asociado de algún modo en ellas a un mecanismo de intransitivización de los verbos transitivos correspondientes, que habrían sufrido, merced a su concurso, un proceso de recesión valencial (Tesnière 1976); es decir, la cancelación de su exigencia paradigmática de complementación directa obligatoria.

La intransitivización operada por SE en los esquemas oracionales que analizamos no es, sin embargo, la única función desempeñada por la forma átona reflexiva en los esquemas ergativos. Estos, aunque intransitivizados, son claramente distintos de otros como (9)-(11), en los que SE originaría — de ahí lo anómalo de (9i-11i) — un proceso similar de bloqueo de la transitividad verbal paradigmática (Martín Zorraquino 1979) que se acomoda literalmente incluso a la propuesta tesneriana sobre la naturaleza de la diátesis recesiva.

(9) el Sr. Lacierva SE apoya en un abominable bastón de cerezo, comprado en la Dalia Azul de Murcia (PUE, p. 54)

(9i) *El Sr. Lacierva SE apoya A SÍ MISMO en un abominable bastón de cerezo, comprado en la Dalia Azul de Murcia

(10) Y cuando ya habían comido todos, SE levantaba... (CONF, p.17)

(10i) *Y cuando ya habían comido todos, SE levantaba A SI MISMO...

(11) ... a una hora fija SE sentaba en el jardín del casino (CONF, p. 16)

(11i) ...*a una hora fija SE sentaba A SÍ MISMO en el jardín del casino

Del carácter escasamente distintivo de los mecanismos de intransitivización como hecho singularizador de las construcciones que analizamos puede también dar idea el hecho de que la intransitivización de cualquier predicado oracional construido con una forma reflexiva átona ha sido postulada incluso como una de las características generales ocasionada por el concurso de estas formas pronominales en cualquier oración ${ }^{3}$. Desde tales planteamientos, sostenidos a veces para justificar la existencia de una supuesta voz media de la que participarían la totalidad de construcciones con SE, las formas reflexivas son

3 Véanse, entre otros, Reichenkron 1933, Oca 1914, Roca 1970, Babcock 1970, Lázaro Mora 1983 o Cano Aguilar 1981. 
concebidas como marcadores que, por su correferencialidad con el sujeto oracional, permiten siempre la presencia en la oración correspondiente de una entidad extensional menos de las funcionalmente operativas.

Es cierto que, desde un punto de vista puramente extensional las formas reflexivas pueden ser concebidas como causantes de un proceso de recesión como el que indicábamos, y que dicho proceso puede ser considerado como una constante en la totalidad de la serie de enunciados oracionales reflexivos. Sin embargo, dicho fenómeno es, como puntualizábamos, de naturaleza extensional; es decir, una característica de la serie en cuestión considerada, no en su dimensión estrictamente endolingüística, sino en virtud de la existencia o no de referentes específicos para unas ciertas formas gramaticales. Al margen de este parecido familiar efectivamente característico de la totalidad de formas reflexivas, no deja de ser sin embargo evidente que tales formas poseen, en su dimensión estrictamente endolingüística, comportamientos funcionales distintos que en modo alguno son ni conectados merced a esta hipótesis explicativa, ni tampoco ilustrados siquiera en la claves de su funcionamiento lingüístico individual.

Desde un punto de vista funcional, la presencia de SE en los enunciados ergativos reflexivos origina una transclasificación de los núcleos verbales correspondientes, que no sólo modificarían este su estatuto transitivo primario - como también sucedería en (9)-(11) - sino también su condición morfosemántica paradigmática. Así, mientras las formas verbales de (9)-(11) tienen en tales oraciones el mismo comportamiento morfosemántico que en enunciados transitivos diferentes como (12)-(14); es decir, constituyen en todos los casos el núcleo funcional de predicaciones de acción, la clase paradigmática e inmutada de los verbos correspondientes, y, como tales, pueden ser a veces, por ejemplo, complementables con indicaciones de finalidad, como se advierte en (9ii)-(11ii) y (12i)-(14i), los esquemas ergativos reflexivos se comportan a este respecto en modo diferente, originándose, merced a la presencia de SE, junto al ya mencionado proceso de intransitivización, una modificación de la clase morfosemántica del verbo en función nuclear-predicativa.

(12) El Sr. Lacierva apoyó la mano sobre el bastón

sujeto agente nuc.pred.acción

(13) El niño levantó sus dos piernas

sujeto nucl.pred

agente acción 
(14) La anfitriona sentó cerca a sus invitados

sujeto agente n.pr.acción

(9ii) El Sr. Lacierva se apoya en un abominable bastón... PARA DESCANSAR SUS FATIGADAS EXTREMIDADES

(10ii) Y cuando ya habían comido todos, se levantaba PARA RETIRAR LOS CUBIERTOS (1 1ii)...a una hora fija se sentaba en el jardín del casino PARA DISFRUTAR DEL SILENCIO

(12i) El Sr. Lacierva apoyó la mano sobre el bastón PARA DESCANSAR MÁS CÓMODAMENTE

(13i) EI niño levantó sus dos piernas PARA GOLPEAR LA PELOTA

(14i) La anfitriona sentó cerca a sus invitados PARA NO PERDER NI UNA DE SUS PALABRAS

Dentro del heterogéneo conjunto de las construcciones reflexivas, el proceso de transclasificación que tiene lugar en las estructuras ergativas reflexivas tendría un cierto equivalente en mecanismos como los que operan en el seno de las oraciones denominadas pasivas reflejas. Enunciados como (15)-(18), pertenecientes a tal tipo, ponen, en efecto, de manifiesto esquemas oracionales de la estructura [sujeto-objeto, núcleo de predicado-proceso...] en los que los papeles morfosemánticos de proceso se incardinan en formas verbales paradigmáticamente especializadas en papeles morfosemánticos de acción o de experimentación, tal y como manifiestan estructuras como (15i), (17i) y (16i), (18i), respectivamente esquemas con la estructura [sujeto-agente, núcleo de predicado-acción...] y [sujeto-experimentador, núcleo de predicado-experimentación...].

(15) Esa noche, mientras SE velaba el cadáver en la gallera José Arcadio Buendía entró en el dormitorio... (CAS, p. 26).

(15i)Esa noche, mientras velaban el cadáver en la gallera, José Arcadio Buendía entró en el dormitorio...

(16) SE ve que está muy solo (CAS, p. 27)

(16i) Ve que ésta muy solo

(17) SE celebran ferias populares como las de Medina del Campo y las de Alcalá (PUE, p. 47)

(17i) Celebran fiestas populares como las de Medina del Campo y las de Alcalá

(18) ...desde allí no SE ven las cosas como las ve el hombreque vive apoyado en la mancera...(CONFE, p. 5)

(18i) ...no ven las cosas como las ve el hombre que vive apoyado en la mancera...

La presencia de SE en las oraciones pasivas reflexivas, además de estar implicada en el proceso de intransitivización que afectaría a todas las estructu- 
ras en las que dicha forma concurre, desencadena muy especialmente un cambio en la estructura diatética de los enunciados correspondientes, que se organizan en tal caso sobre el eje oracional de un núcleo del predicado de proceso, y no ya de acción o experimentación, como es característico de las respectivas formas verbales no marcadas mediante el átono reflexivo.

El carácter pasivo de oraciones como (15)-(18) no puede ser, sin embargo, vinculado exclusivamente a esta estructura morfosemántica oracional. Enunciados como (19) o (20), con la estructura de relaciones sintácticas [sujeto-objeto, núcleo de predicado-proceso], no pueden ser adecuadamente descritos como oraciones con significación pasiva alguna, y ello en razón de que está «pasividad» resulta, en estructuras como las de (15)-(18), de la conjunción de las relaciones ya mencionadas con otro rasgo característico: la existencia funcional de un papel sintáctico de agente o experimentador, que indican en ellas precisamente cómo el proceso que se predica del objeto es producido por un agente, como en (15) y (17), o afecta a un experimentador, como en (16) y (18).

(19) El telón cayó

[sujeto-objeto, núcleo del predicado-proceso]

(20) Pedro resbaló

[sujeto-objeto, núcleo del predicado-proceso]

Como se ha apuntado (Vera Luján 1990), en las denominadas oraciones pasivas reflejas los papeles de agente o experimentador correspondientes constituyen otras tantas relaciones morfosemánticas genéricas o indeterminadas, señaladas formalmente precisamente por la presencia de la forma átona refleja de tercera persona. Se trata del mismo papel genérico que merced al concurso de dicha forma opera en las oraciones habitualmente denominadas impersonales reflejas, oraciones como (21)-(23), que deberían ser, más adecuadamente, caracterizadas como oraciones de sujeto genérico o indeterminado, y en las que encontramos, en efecto, estructuras con sujetos que pueden ser clasificados como pertenecientes a los papeles morfosemánticos de agente, (21i), experimentador, (22i) u objeto, (23i), pero en todos los casos de agentes genéricos, experimentadores genéricos y objetos genéricos para cuya denotación nuestra lengua, al no poseer signos lingüísticos específicos capaces de significarla, utiliza parcialmente la forma átona refleja de tercera persona (Vera Luján, 1990), que carece, por lo demás, en estas oraciones de efecto transclasificador alguno sobre la dimensión paradigmática de las formas verbales correspondientes, especializadas primariamente en funciones temáticas de acción, experimentación y estado, que cumplen, respectivamente, en (21)-(23) exactamente igual que en enunciados similares, como (21ii)-(23ii), carentes de SE y con sujetos especificados: 
(21) ...se me amonestaba porque volvía tarde. (CONF, p. 18)

(22) ... hay en ellos ratos interminables en que no se sabe qué hacer. (CONF, p.

18)

(23) Se está siempre cerca de una greguería, pero no se la toca (FLOR, p. 18)

(21i) se me amonestaba porque volvía tarde

Agente gco. Acción

(22i) $\ldots$ no se sabe qué hacer

Experimentador Experimentación genérico

(23i)

Se está siempre cerca de una greguería...

Objeto gco. Estado

(21ii) Mi madre me amonestaba porque volvía tarde

Agente Acción

(22ii) El profesor no sabe qué hacer

Experiment. Experimentación genérico

(23i) Todo el mundo está siempre cerca de una greguería...

Objeto Estado

El papel cumplido por SE en las estructuras ergativas reflexivas difiere de su función en los esquemas oracionales mencionados. Mientras en los últimos su presencia se asocia a la indeterminación de ciertos papeles temáticos, en aquéllas produce, al contrario, una auténtica transclasificación nuclear-predicativa, originando que formas verbales de un funcionamiento paradigmático tal que han de construirse necesariamente con el concurso del papel morfosemántico de agente, eliminen tal relación de entre las que constituyen su estructura de valencias ${ }^{4}$. Así, mientras verbos como torcer, alegrar, alarmar..., que concurren en los enunciados que analizamos, exigen en sus empleos no-marcados la valencia agentiva que aparece en ejemplos como (1iii)-(3iii) materializada en la función de sujeto, en (1iv)-(3iv) en la de circunstancial agentivo, o con una

4 Véase J. Andrés de Molina (1974). 
significación de máxima indeterminación señalada por la presencia de SE en las oraciones pasivas reflexivas ejemplificadas en (1v) o (6iii), su concurso en los esquemas oracionales ergativos reflexivos supone la cancelación de dicho papel agentivo, como muestra la imposibilidad de paráfrasis de los enunciados citados dotadas de sentido ergativo cuando se mantiene la presencia de complementaciones típicamente agentivas como las de finalidad - (1 vi)-(3vi) , o concurren complementaciones instrumentales que también requieren la presencia estructural de una figura agentiva en el enunciado correspondiente (1vii) y (3vii)-.

(1iii) ... el herrero torcía bisagras y aldabas con su fuerza descomunal (2iii) El circo alegraba a todo el pueblo

(3iii) El estruendo alarmó a todos

(liv) ... bisagras y aldabas eran torcidas por el herrero...

(2iv) Todo el pueblo era alegrado por el circo

(1v) bisagras y aldabas se torcían con ningún esfuerzo

(6iii) El tren se ha puesto otra vez en marcha sin prestar atención

(1vi) *bisagras y aldabas se torcían de calor para que se acomodasen a las medidas del carpintero

(2vi)...*El pueblo se alegraba con los acordes de una misma pieza para celebrar la llegada del verano

(3vi) *Al principio nadie se alarmó para asustarse

(1 vii) *bisagras y aldabas se torcían de calor con unas inmensas tenazas (3vii) *AI principio nadie se alarmó con su historia

Salvadas estas diferencias que tienen que ver con la cancelación del papel temático agentivo en los enunciados reflexivos ergativos, éstos se asemejan, como indicábamos, a los denominados pasivos reflejos por su condición de enunciados con núcleos predicativos procesuales; es decir, equivalentes a otras tantas funciones sintácticas oracionales de carácter dinámico, a diferencia de los estados, y que indican acontecimientos no agentivos, a diferencia de las acciones (Dik 1978).

La significación procesual de los núcleos predicativos de las estructuras ergativas reflexivas es lo que, precisamente, caracteriza a estas oraciones frente a otras como (24)-(25), en ocasiones denominadas medias (Keyser-Roeper 1984, Zubizarreta 1987, Demonte 1989) en las que, si bien se produce, merced al concurso de $\mathrm{SE}$, un proceso de cancelación valencial similar al ya reseñado -véanse (24i)-(25i) - y un mecanismo paralelo de transclasificación nuclearpredicativa, este último es de tal naturaleza que los verbos utilizados en dicha función pasan a denotar estados o propiedades del sujeto; predicaciones, por 
tanto, no desarrolladas a lo largo de un determinado periodo de tiempo - de donde lo anómalo de (24ii) o (25iii) como hipotéticos sentidos estativos; es decir, como cualidades características de un determinado 'preparado' o del 'hielo'-:

(24) Este preparado se funde con facilidad

(24i) El calor funde este preparado con facilidad

(24ii) *Este preparado se está fundiendo con facilidad

(25) El hielo se derrite

(25i) El calor derrite el hielo

(25ii) *El hielo se está derritiendo

Como ya hemos indicado, en los esquemas oracionales que analizamos la significación procesual de los núcleos predicativos se corresponde con formas verbales paradigmática o primariamente especializadas en papeles diferentes los de acción-, y propias de esquemas oracionales también distintos: primariamente los de [agente-acción], como se muestra en los enunciados que a continuación se indican:

(1iii) El herrero torcía bisagras y aldabas con su fuerza descomunal

(2iii) El circo alegraba a todo el pueblo

(3iii) El estruendo alarmó a todos

(4vii) El calor agostó las mieses

(5vii) La muerte de su amigo le afligió profundamente

(6vii) El maquinista ha puesto en marcha otra vez el tren

Las formas verbales propias de los esquemas oracionales de [agente-acción], torcer, alegrar, alarmar, agostar, poner en marcha..., concurren en los esquemas ergativos reflexivos con la significación de proceso en virtud, fundamentalmente, de la presencia de SE, que está, pues, claramente asociada al mecanismo de transclasificación que se desarrolla:

(1 viii) *... bisagras y aldabas torcían de calor

(2vii) *... el pueblo alegraba con los acordes de una misma música

(3viii) *Al principio nadie alarmó

(4viii) *Las mieses también han agostado por falta de Iluvias oportunas

(5viii) *...hubo un pequeño viejo, mi bisabuelo, que afligió profundamente

(6viii) *El tren ha puesto otra vez en marcha...

Como último aspecto descriptivo que merece la pena destacar en las estructuras ergativas reflexivas, éstas presentan en la posición de sujeto sintagmas que contraen las funciones temáticas de experimentador - (3) y (5) - o de objeto - (1),(2), (4) y (6)—; es decir, nombres que se comportan o funcionan 
como entidades capaces de ciertos cambios psicológicos o como meras entidades afectadas por los procesos, respectivamente. Especialmente en lo que a los segundos se refiere, los enunciados ergativos reflexivos muestran la particularidad, reseñada en distintas ocasiones (Moreno 1984, Demonte 1991), de admitir tan sólo el concurso de una entre las distintas subclases de la relación morfosemántica objeto, el denominado objeto afectado; es decir objetos relativamente independientes de las operaciones significadas por el núcleo predicativo, nunca producidos como su resultado, tal y como, por ejemplo, sucedería en (26)-(28), cuyos complementos directos han de ser concebidos como objetos resultativos; es decir entidades no existentes sino como resultado y a continuación de la 'acción' verbal, y por lo mismo no admitidas como sujetos en las estructuras ergativas reflexivas:

(26) Los niños imaginan historias truculentas

(26i) *(Las) historias truculentas se imaginan

(27) Fabricó un mueble hermosísimo

(27i) *Un mueble hermosísimo se fabricó

(28) Pensaban una magnífica solución

(28i) *Una magnífica solución se pensaba

En conclusión, los esquemas oracionales que denominamos ergativos reflexivos constituyen una serie de estructuras oracionales cuyos rasgos funcionales más significativos son los resumidos en (29):

(29)

i) núcleo predicativo de proceso

ii) sujeto de papeles temáticos experimentador/objeto afectado.

iii) incompatibilidad con el papel temático agente y complementaciones propias.

iv) estructura funcional-oracional marcada por la forma átona reflexiva SE+formas verbales paradigmáticamente agentivas.

(29) puede, deseablemente, ser considerada como una adecuada descripción de las peculiaridades más relevantes del funcionamiento de las estructuras ergativas reflexivas. Supone, esencialmente, una caracterización de tales oraciones que las categoriza como una subclase de entre las que forman parte de la clase más general de los esquemas oracionales procesuales en español, y da cuenta, además, de cómo los esquemas ergativos reflexivos tienen, tomando en consideración el material léxico en que se incardinan, una singularidad especial: un carácter procesualmente secundario, al ser construidos, merced a un mecanismo de transclasificación, a partir de formas paradigmáticamente agentivas.

Como caracterización puramente descriptiva, (29) no incluye, sin embargo, ninguna hipótesis explicativa sobre algunas cuestiones de innegable interés 
lingüístico o metalingüístico: fundamentalmente, si es posible establecer mecanismos generales que permitan explicar motivadamente los procesos de transclasificación nuclear-predicativos y, más específicamente, los mecanismos que están involucrados en la transformación de los papeles temáticos predicativos acción $\rightarrow$ proceso merced al concurso del átono SE.

No es, naturalmente, éste el lugar para abordar en toda su presumible complejidad una teoría general de la transclasificación sintáctica; ni siquiera para desarrollar en todos sus detalles la más limitada que consideraría las claves generales a que obedecerían los mecanismos de transclasificación predicativa, pero, a la luz de determinados planteamientos relativamente recientes sobre el modo en que puede ser concebida la representación de la estructura semántica de los predicados, sí creemos posible delimitar una imagen de los procesos de transclasificación que afectan a las oraciones que estudiamos como resultado, no de reglas puramente arbitrarias, sino de transformaciones que encontrarían en las peculiaridades de la forma SE y en la misma estructura semántica de ciertos predicados agentivos una motivación evidente.

De manera muy simplificada, podemos concebir en sus aspectos más generales la estructura de un modelo gramatical adecuado como un simulacro que relacione los componentes oracional y léxico mediante determinadas operaciones o principios de articulación que establecerán, básicamente, cómo las distintas funciones sintácticas oracionales son incardinadas en cierto material léxico, compatible con dicho funcionamiento ${ }^{5}$. Es también posible, en el sentido en que distintos modelos lingüísticos formales lo vienen postulando ${ }^{6}$, concebir el lexicón de una lengua no sólo como un mero inventario de propiedades irrepetibles, sino como un componente lingüístico que albergaría también regularidades entre las que se contarían las que formalizaría el componente morfológico, que a partir de un mismo material léxico de base daría origen a distintos elementos léxicos terminales.

En un modelo de tales características generales, el lexicón constituye el componente gramatical en el que se hallarían especificaciones léxicas y gramaticales de los distintos elementos léxicos de una lengua; aquellas propiedades que los singularizan como formas concretas y que deben, por consiguiente, ser conocidas por los hablantes para poder utilizar adecuadamente las formas en cuestión. En el ámbito específico del comportamiento sintáctico-oracional que nos ocupa, tales propiedades habrían de consistir, según es ya planteamiento habitual en buen número de modelos lingüísticos actuales, tanto en los aspectos constitutivos de las relaciones sintácticas oracionales (funcionamiento como

5 Adoptamos en este punto una visión laxa que busca aproximar puntos de vista diferentes en detalle, incluidos los más recientes de la gramática generativa y las gramáticas cognitivas o conceptuales, en el sentido de que la generación oracional se produce ya desde el mismo léxico.

- A partir ya del clásico trabajo de Chomsky (1970). 
sujeto, núcleo de predicado, complemento directo..., etc.), como también en las dependencias (Báez 1988) a que dichas relaciones están sometidas en el marco oracional, especialmente bajo la forma de lo que se denominan casos (Fillmore 1968) funciones semánticas (Dik 1978), relaciones temáticas (Gruber 1976), papeles temáticos (Dowty 1986), etc.

Aceptando, pues, que nociones como las mencionadas juegan un papel básico en la modelización del funcionamiento de las estructuras sintácticas oracionales de una lengua, en el marco más general reseñado de la estructura de la gramática de una lengua, es posible esquematizar, a muy grandes rasgos, el funcionamiento en estos aspectos de un modelo lingüístico como la incardinación de ciertas relaciones sintácticas oracionales constitutivas y de dependencia en un material léxico, suministrado por el lexicón a través de su componente propiamente léxico o a través del morfológico, compatible con estas relaciones.

Con tales planteamientos, muy resumidamente, toda forma verbal recibiría en el lexicón una definición que, además de especificar otras propiedades, incluiría, junto a la de su capacidad constitutiva para funcionar como núcleo del predicado oracional, la de sus posibles dependencias; relaciones estas que, de ser categorizadas en la manera en que, como venimos viendo, es habitual, operarían con las clases de verbos/predicados mencionadas de estados, acciones y procesos (Dik 1978); los primeros caracterizados por el rasgo [+estático], acciones y procesos por el de [-estático]. Estos últimos diferenciaría, respectivamente, entre acontecimientos que suponen un obrar (y que, característicamente originan oraciones con sujetos agentes) y aquellos otros reducidos a la mera significación de una acontecer que afecta a ciertas entidades (en las oraciones correspondientes, con sujeto temático objeto o experimentador).

La utilización de las clases de relaciones de dependencia nuclear-predicativas mencionadas haría imposible, sin embargo, el empeño de establecer una regla morfológica motivada que formalizara la relación advertida entre verbos como los de (1)-(6) y los correspondientes verbos carentes de la forma SE, toda vez que dicha relación habría de ser formulada como consecuencia de un cambio del tipo proceso $\leftarrow$ acción, dos clases primitivas definidas sin relación alguna entre sí, más allá de su común condición no estativa. Junto a esta perspectiva en la definición de los papeles temáticos mencionados, hay, no obstante, otro punto de vista que parece constituir hoy la orientación más importante por lo que se refiere al estudio de las relaciones temáticas predicativas, especialmente en el ámbito de las gramáticas generativas, y que ha sido desarrollado en distintos trabajos (Kenny 1963, Vendler 1967, Dowty 1979) que postulan la existencia de una dimensión aspectual esencial en el funcionamiento de los predicados oracionales. Más específicamente, algunos de estos trabajos (Vendler 1984, Grimshaw 1992, Demonte 1989) sugieren —en la línea de planteamientos similares propios de la denominada «semántica generativa»- que la significación morfosemántica o temática de los predicados oracionales puede ser conce- 
bida como una estructura compleja eventiva con cuyos elementos constitutivos se relacionan sintácticamente los distintos elementos-funciones oracionales.

Vendler (1984) ha propueto, en esta línea, considerar los predicados de acción como predicados con una estructura compleja como la esquematizada en (30), en la que han de distinguirse distintas posiciones funcionales, que podríamos traducir como HACER (do), CAUSAR (c) y EVENTO (event), las cuales gobernarían distintos elementos oracionales: en los enunciados de (31)-(33) respectivamente a easily, carefully y fast.

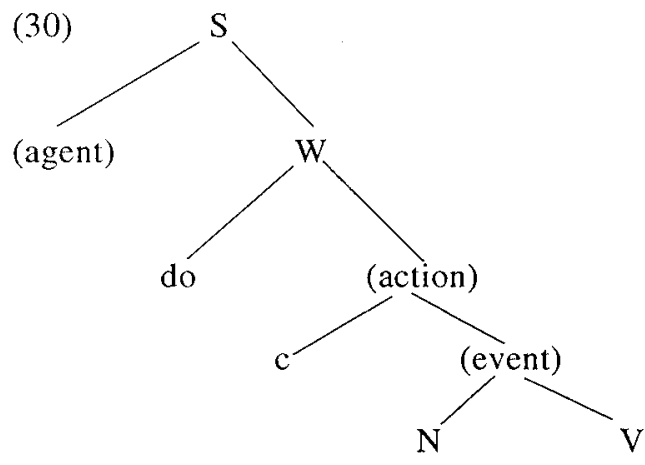

(31) He drove the car fast

(32) He drove the car carefully

(33) He drove the car easily

Grimshaw (1992) ha mostrado lo insatisfactorio de una descripción de la estructura de la oración basada exclusivamente en las dimensiones sintácticoconstitutiva y temática, postulando, al contrario, la necesidad de su caracterización en términos de dos jerarquías de argumentos, una temática y otra aspectual. Esta última tomaría la forma de lo que denomina «event structure» y determinaría, en último extremo, la estructura combinatoria oracional, pues los distintos constituyentes no verbales de la oración vendrían a estar gobernados por alguno de los componentes aspectuales de dicha «estructura eventiva», cada uno de cuyos subcomponenteș semántico-aspectuales habría de encontrar su manifestación en alguno de dichos elementos. En la propuesta de Grimshaw, su análisis «eventivo» daría origen a la tipología de predicados indicada en (34), en la que se observan, en efecto, ciertos tipos predicativos con una estructura de subcomponentes aspectuales, cada uno de los cuales, como indicábamos, gobernaría a determinados elementos «nominales» de la oración. Así, para enunciados pasivos como (35) habría de suponerse una estructura eventiva compleja 
como (36), en la que existiría un subcomponente eventivo de actividad que gobernaría las complementaciones agentivas obligatorias en esta oración, ya que la supresión de tales complementaciones originaría enunciados anómalos.

i) realización

[[actividad] [estado/ cambio de estado]

ii) inergativo

[actividad]

iii) [inacusativo]

[estado/cambio de estado]

(35) This house was built/designed by a French architect

(35i) *This house was built/designed

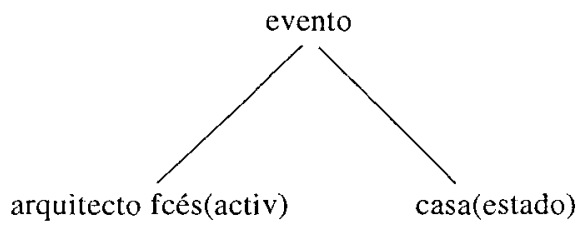

En la línea de planteamientos indicada, Demonte (1989) ha destacado la rentabilidad explicativa de estas hipótesis para la caracterización de las estructuras ergativas reflexivas, postulando, en el marco de los análisis clásicos de Keyser y Roeper (1984), su condición anticausativa y considerándolas como oraciones en las que «desde el punto de vista aspectual, el único componente del evento es el resultado» (Demonte, 1989; 101).

La consideración de los predicados oracionales como estructuras semánticoeventivas complejas implica, en consecuencia, la caracterización de los predicados realizativos de oraciones como (1iii)-(3iii) en una forma similar a (37); es decir, como fórmulas semánticas complejas estructuradas en un subcomponente semántico-eventivo de actividad y otro de cambio de estado, o proceso, cada uno de los cuales gobernaría funcionalmente a determinados elementos actanciales: el subcomponente de actividad al papel temático agentivo que aparece como sujeto en (1iii)-(3iii) o como circunstancial agentivo en (1iv) y (2iv), o a los 
circunstantes de (1vi)-(3vi); el subcomponente de proceso al papel objeto afectado/experimentador que encontramos en función de complemento directo en (1iii)-(3iii) o de sujeto en las estructuras ergativas reflexivas -recuérdense (1)-(6) - De otro lado, la categorización de los predicados agentivos como estructuras de evento complejas de la forma de (37) permite, en efecto, plantear la existencia de una relación evidente entre determinadas oraciones con predicados realizativos y las ergativas reflexivas que analizamos. Éstas presentarían, en este esquema explicativo, predicados cuya significación procesual es sólo uno de los dos subcomponentes de la estructura de eventos más compleja de las oraciones de realización-(38)- En tales términos, el proceso de transclasificación que ciertos predicados realizativos sufren para convertirse en predicados de proceso resulta, finalmente, consecuencia de una operación de supresión, en sus aspectos más generales idéntica, como tal mecanismo lingüístico-general, a otros procesos de supresión que es posible rastrear en distintos componentes de la gramática de las lenguas.

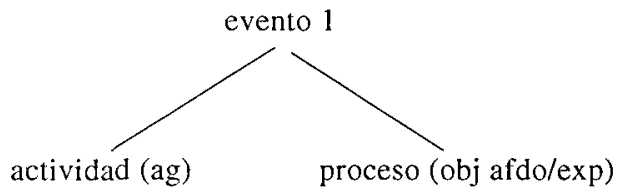

(38)

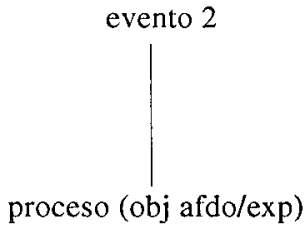

Aunque la explicación del proceso de transclasificación realización $\rightarrow$ proceso como producto del cambio de una determinada estructura eventiva dotada de dos subcomponentes constituyentes a otra en la que se conserva uno solo de 
ellos presenta al mecanismo de transclasificación en cuestión como un proceso claramente motivado, queda aún una interrogante evidente por responder para poder considerar razonablemente resuelta la tarea de explicar la relación entre las estructuras de acción y las ergativas reflexivas que analizamos: la del papel cumplido por el átono SE en dicho proceso como constituyente obligatorio de una hipotética regla morfológica del tipo de (39), que permitiría obtener verbos de proceso a partir de otros de realización de manera plenamente productiva.

$V$ con la estructura semántico-eventiva [ag(actividad) obj afdo/exp(proceso)] $\rightarrow \mathrm{V}+\mathrm{SE}$ [obj afdo/exp (proceso)]

En realidad, como intentaremos mostrar, el papel desempeñado por SE en (39) es el esperable, si tenemos en cuenta el comportamiento de este elemento en algunos de sus empleos no prototípicos, como son los que caracterizan a esta forma en las denominadas oraciones pasivas o impersonales reflexivas, y tomamos paralelamente en consideración los que serían los principios generales por los que se rige la articulación de los componentes léxico y oracional de las lenguas.

La articulación de los niveles oracional y léxico de una lengua puede concebirse, simplificadamente, como un proceso por el que las relaciones sintácticas oracionales que subyacen a un enunciado son incardinadas en cierto material léxico suministrado por el diccionario de una lengua (ya sea a partir de su subcomponente propiamente léxico o del morfológico). Las relaciones sintácticas que afectan a los núcleos predicativos de los enunciados oracionales tienen que ver, en lo que aquí nos interesa, con dos grandes grupos de hechos: los que podemos denominar su valencia específica $\rightarrow$ o conjunto de relaciones que caracterizan individualmente a un núcleo predicativo-y su valencia genérica -las relaciones de constitución que se incardinan bajo la forma de circunstantes de distintas clases temáticas y que caracterizan a determinados tipos de estructuras oracionales con las que son característicamente compatibles, mientras que son excluidas por otras-. Formaría parte, por ejemplo, de la valencia específica de un núcleo predicativo que incluya el subcomponente de actividad la exigencia de construirse con sujetos agentes; sería característico de la valencia específica de los inacusativos el exigir sujetos en funciones temáticas de objeto o experimentador..., etc. Dependería de lo que hemos denominado la valencia genérica, al contrario, la propiedad de los predicados que presentan el subcomponente de actividad de construirse con circunstanciales de finalidad o instrumento, que pueden o no apdrecer en algunos enunciados de estas características, pero que están vedados en otros esquemas como los de núcleos inacusativos.

En lo relativo a los factores que tienen que ver exclusivamente con las relaciones sintácticas oracionales, la articulación de los componentes oracional 
y léxico consistiría en la incardinación de las relaciones oracionales mencionadas en un material léxico con las mismas propiedades, oportunamente especificadas en las distintas entradas léxicas o en las reglas morfológicas correspondientes del diccionario.

Dentro de este esquema general, la articulación de los dos niveles gramaticales que consideramos parecería responder a una serie de principios como los enumerados en (40):

i) se incardinan en una estructura oracional las formas léxicas predicativas que coinciden en sus valencias con las relaciones funcionales actualizadas en el enunciado oracional correspondiente.

ii) La valencia genérica de un verbo puede o no formar parte del enunciado; la valencia específica debe satisfacerse siempre.

iii) Las valencias específicas de un verbo pueden anularse. En tal caso, debe existir un significante asignable a la relación anulada.

(40) esquematiza, en efecto, unos cuantos principios muy generales que parecen cumplirse necesariamente en los casos en los que los procesos de inserción léxica se desarrollan satisfactoriamente. (40i) enuncia sin duda la exigencia más elemental y transparente de que ninguna forma verbal puede hacerse servir para papeles que no entran en sus privilegios de figuración oracional. Así, por ejemplo, un verbo como comer no podría ser insertado en la posición de núcleo predicativo de un esquema impersonal pues se trata de una forma en cuya valencia específica está la exigencia de construirse con sujeto.

(40ii) alude a los hechos bien conocidos de que, mientras las complementaciones circunstanciales propias de una clase de oraciones, aun caracterizándola estructuralmente por su presencia siempre posible, no necesariamente han de actualizarse en un concreto esquema oracional, la que llamamos valencia específica es de aparición inexcusable: así, por ejemplo, mientras circunstantes instrumentales como el de (41) pueden aparecer o no en enunciados en los que está presente el (sub)componente de actividad (mientras que no son admisibles con dicho papel temático sintagmas semejantes en enunciados con el de estado — véase a este respecto (42)—), el sujeto agente de comer es estructuralmente obligatorio.

(41) Cierra la puerta (con la llave)

(42i) *Es cerrajero con la llave

(40iii), por su parte, alude a la posibilidad de que determinadas relaciones específicas sean eliminadas de entre las exigencias de complementación de una forma verbal. Establece como principio el hecho bien conocido de que la eliminación de una valencia de esta naturaleza no se logra simplemente no 
actualizando ninguna forma léxica destinada a desempeñar dicha función, sino que es precisa alguna operación de signo positivo para señalar expresamente la cancelación efectuada. Así, por ejemplo, la no existencia en (41) de un sintagma al que poder adjudicar la función de sujeto oracional no convierte a (41) en una estructura impersonal, posibilidad que requiere de determinadas transformaciones siempre acompañadas de sus correspondiente marcas significantes.

En este marco metodológico general, esbozado muy superficialmente por razones evidentes, el papel de SE como forma capaz de operar el mecanismo de transclasificación indicado en (39) es, como adelantábamos más arriba, explicable en la línea de lo que son los comportamientos no prototípicos de dicha forma.

Como Vera (1990) mostró, la categoría 'reflexivo átono de tercera persona' puede ser estructurada internamente desde la perspectiva de la denominada teoría de prototipos, distinguiendo entre el prototipo, o miembro especialmente representativo de la categoría, cuyas características -que corresponden a los empleos funcionales de oraciones como (7) u (8) - se recogen en (43), y otros miembros no prototípicos, que suponen lo que Lakoff (1977) denominara utilizaciones parciales de la categoría, la posesión tan sólo de ciertas propiedades del conjunto de las que caracterizan al prototipo categorial.

(43) ${ }^{7}$ i) Morfema

ii) Deíctico

iii) $\mathrm{OE}$ +animado

iv) miembro del paradigma pronominal reflexivo: indicador de

concordancia con complemento directo/indirecto.

v) referencia coincidente con la del sujeto oracional

vi) dominio de referencia en su oración

Entre estos comportamientos no prototípicos sin duda los más frecuentemente tomados en consideración son los que se advierten en las llamadas oraciones

7 Respecto de los rasgos utilizados en la definición del prototipo, (43i) indica que se trata de un elemento incapaz de actualización autónoma y limitado por ello a una funcionalidad morfemática que le liga siempre al verbo para su actualización en un enunciado. (43ii) destaca que SE es una forma de significación deíctica cuyo contenido correspondería, en términos hegerianos (Heger 1974), a la dimensión personal-comunicativa, dentro de la cual se definiría como forma $O$, es decir que no constituye el punto inicial del circuito comunicativo $-\mathrm{O}-$, y $\mathrm{E}$, es decir, no corresponde a un participante directo en la relación comunicativa - E-; destacándose, además, su carácter necesariamente [tanimado]. (43iv) refleja que $\mathrm{SE}$ es, como todas las formas átonas pronominales, un elemento esencial en la constitución de los mecanismos de concordancia que identifican a las relaciones funcionales de complemento directo e indirecto, al reiterar en el verbo indicaciones también contenidas en los sintagmas que desempeñan dichas funciones (Vera Luján 1990). El resto de propiedades enumeradas destacan ciertas propiedades esenciales de las formas reflexivas; básicamente, el hecho de que se trata en todos los casos de formas carentes de capacidad referencial autónoma y que obtienen dicha referencia de la obligada identidad entre el complemento directo/ indirecto y el sujeto. 
pasivas e impersonales reflexivas, en las que SE presenta, en efecto, características de funcionamiento que nada tienen que ver con algunas de las propiedades caracterizadoras del prototipo. Así sucede con los rasgos definidos en (43iv-vi) que carecen de funcionalidad alguna en oraciones como (15) o (21), donde SE no participa en ningún mecanismo de concordancia objetiva entre el núcleo del predicado y unos supuestos complementos directo o indirecto, ni, en consecuencia, marca la característica identidad referencial prototípica entre el sujeto y tales funciones complementarias. Las únicas propiedades de (43) que parecen convenir al funcionamiento de $\mathrm{SE}$ en (15) o (21) son las reseñadas como (43i-iii), que estarían asociadas a la indeterminación de ciertos papeles sintácticos característica de las oraciones consideradas; a la indeterminación del sujeto de las estructuras como (21) o a la de ciertos circunstantes en las del tipo de (15).

$\mathrm{La}$ indeterminación de estas relaciones funcionales es marcada, fundamentalmente, merced a las propiedades enumeradas de SE, por razones que pueden ser explicadas en la línea de una propuesta como la de Vera (1990). Por lo que a los enunciados denominados comúnmente impersonales reflexivos_ se refiere, éstos son la manifestación de estructuras oracionales como (44), en las que las relaciones sintácticas constitutivas de sujeto y núcleo predicativo se organizan bajo la forma de diversas clases de dependencias temáticas ${ }^{8}$; así, por ejemplo, en (21), las de agente y —utilizando la tipología de Grimshaw explicitada en (34) - realización, respectivamente. Es rasgo fundamental de enunciados como (21), además, el que se construyan como enunciados en los que la relación sujeto-agente se desea materializar léxicamente como incardinada en un elemento de la máxima indeterminación, o de la condición genérica más acentuada. Teniendo en cuenta, de un lado, que el español carecería de elementos léxicos «nominales» adecuados con dicha significación, o de formas verbales con una valencia específica de tales características, y, de otro, que, según establecimos en (40ii), la posición de sujetoagente no puede ser dejada 'vacía', la utilización parcial de SE constituye, finalmente, la única alternativa posible para señalar la especial condición semántica de los sujetos en cuestión; una indeterminación que SE puede señalar como elemento de significación no simbólica, sino meramente deíctica, y máximamente inespecificada (OE), toda vez que la especificación habitual en su caso, lograda merced a la prototípica identidad referencial sujeto-complemento directo/indirecto, no es de aplicación en esta ocasión.

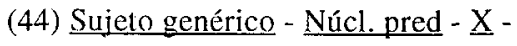

8 Vera (1990) ha mostrado cómo los papeles temáticos que pueden encontrarse en función de sujeto de estas oraciones son los de agente, experimentador y objeto. En cualquier caso, la denominación de impersonales no resulta muy adecuada para la designación de tales oraciones, toda vez que lo que las caracteriza no es en modo alguno la no existencia de la función sujeto, sino el hecho de que dicha función es incardinada en una significación relativa a entidades humanas de la máxima indeterminación. 
En líneas generales, la funcionalidad cumplida por SE en los enunciados pasivos reflexivos es explicable de manera semejante. Se trata en este caso de enunciados que materializan estructuras de núcleo predicativo de proceso y sujeto que funciona temáticamente como objeto, pero con la singularidad de que concurre en ellas un elemento-relación agente o experimentador máximamente genéricos o indeterminados que, de materializarse, habrían de ocupar posiciones funcionales constitutivas de complemento circunstancial":

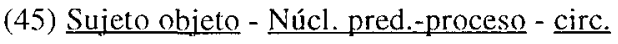 agente/experimentador genéricos}

La conjunción SE+verbo con una estructura de valencias específicas de agente o experimentador constituye también en esta ocasión la alternativa para la materialización de un esquema de tales características: no existiendo, como ya apuntábamos, elemento léxico alguno específico para la significación de los contenidos de máxima indeterminación en nuestra lengua, la utilización parcial del prototipo (43) en sus rasgos (i-iii), en conjunción con la forma verbal elegida, se convierte en significante adecuado de las características sintácticosemánticas mencionadas: por una parte, en efecto, determinadas valencias genéricas verbales, como las que, por ejemplo, caracterizan a las estructuras en las que concurre una relación temática agentiva (finalidad, instrumento...) y que se manifiestan —o pueden hacerlo- en los enunciados correspondientes, señalan la existencia de dichos papeles temáticos, mientras que la utilización parcial del prototipo SE en los rasgos mencionados indica inequívocamente la imposibilidad de especificar referencialmente la identidad de dicho papel que, por consiguiente, resta indeterminado.

Los enunciados reflexivos pasivos y los comúnmente denominados impersonales tienen, pues, en común el que son manifestaciones de estructuras en las que una función oracional corresponde a un elemento léxicamente caracterizado por ser de la máxima indeterminación. Por consiguiente, se trata de enunciados en los que SE no cumple función transclasificadora alguna: son enunciados en que se cumplen las características de las correspondientes formas verbales paradigmáticas, cuya condición morfosemántica no es alterada, y cuya única especificidad consiste, como indicábamos, en esta singularidad léxica.

La función de SE en las estructuras reflexivas ergativas es, como ya se apuntó, diferente, puesto que su presencia es determinante en el proceso de transclasificación verbo realizativo $\rightarrow$ verbo de proceso que es característico de estas oraciones. No obstante, si la función de SE es diferente, creemos que las

9 De ahí la existencia de enunciados no normativos en los que está presente un sintagma agentivo del tipo por... 
claves de este funcionamiento son semejantes a las ya reseñadas. Tienen que ver, en efecto, con la utilización parcial del prototipo SE en los enunciados ergativos reflexivos con el mismo conjunto de propiedades - (43i-iii) - actualizado en las oraciones pasivas e impersonales reflexivas, y también como en ese caso con la inoperatividad del resto de propiedades del prototipo.

Es evidente la inoperatividad en las oraciones ergativas reflexivas de las propiedades del prototipo reflexivo que indican su condición de marca de concordancia núcleo predicativo-complemento directo/indirecto, y de la coreferencialidad sujeto-complemento directo/indirecto. Las únicas propiedades del prototipo SE compatibles con su uso en (1)-(6) resultan, en efecto, las que representábamos en (43i-iii): la condición de SE de elemento funcionalmente no autónomo, su significación deíctica y su carácter de elemento máximamente inespecificado [+animado]; propiedades todas que ofrecen una clave explicativa de su funcionalidad transclasificadora.

En el nivel oracional los enunciados ergativos reflexivos se corresponden con una estructura funcional de la forma de (46).

(46) Sujeto Núcleo pred.

Obj.afdo/exp. Proceso

Según indicáramos, las funciones oracionales de (46) requieren, para su adecuada materilización lingüística (para el paso de su condición de elementos virtuales de un esquema estructural oracional a la de entidades de un enunciado realmente actualizado), de su incardinación en un material léxico caracterizado por poseer propiedades funcionales semejantes.

Específicamente en lo relativo a las formas verbales que pueden concurrir en ellos, los enunciados ergativos reflexivos requieren en posición nuclear predicativa verbos de proceso compatibles con las relaciones temáticas de objeto afectado o experimentador, como se corresponde con la condición temática de los respectivos núcleos predicativos oracionales. Hay verbos, como caer o bajar por ejemplo, cuya valencia paradigmática les faculta directa e inmediatamente para concurrir en estructuras con las propiedades de (46); es decir, en enunciados que, como (19) o (20), corresponden a oraciones con la estructura de relaciones funcionales sujeto-objeto, núcleo predicativo-proceso, y que carecen de papel agentivo alguno. Otros verbos, sin embargo, como los encontrables en las oraciones ergativas reflexivas poseen una estructura eventiva realizativa compleja y cuentan entre sus valencias con la específica de agente, propiedades que determinarían para ellos dos series de posibilidades paradigmáticas de actualización: las activas que se manifiestan en oraciones como (21ii) o (21), con sujeto agente específico o indeterminado según el caso, y las pasivas, reflexivas como (15) o analíticas como (15ii), en las que el papel agentivo es 
virtual/actualmente indeterminado o concreto, respectivamente.

(15ii) ... el cadáver era velado en la gallera...

En el caso de los verbos dotados de una estructura valencial como la indicada, su utilización en esquemas como (46) es necesariamente resultado de distintas operaciones sobre una capacidad de relación estructural que ha de ser modificada para apartarla de sus mecanismos de articulación regulares, merced a la cancelación de la valencia agentiva paradigmática, así como la del subcomponente eventivo de actividad consustancial, según la caracterización de Grimshaw, a los verbos/predicados de realización.

Si adoptamos los planteamientos de Grimshaw en el sentido de que la estructura eventiva verbal/predicativa gobierna a las restantes funciones oracionales, y de que existe una suerte de interdependencia entre subcomponentes eventivos y funciones no nuclear-predicativas, parece claro que podemos suponer que la cancelación de la valencia de agente es una operación interdependientemente asociada a la anulación del subcompoente eventivo actividad, por lo que, en consecuencia, las operaciones canceladoras del papel temático agentivo implicarán solidariamente la anulación del componete eventivo que le gobierna.

En los enunciados ergativos reflexivos el papel de SE puede ser caracterizado, precisamente, como el del elemento que señala la anulación del elementorelación agentivo; una operación que se logra al incardinar la función nuclearpredicativa procesual en verbos que, con un contenido eventivo realizativo, incorporan parcialmente las propiedades (43i-iii) del monema SE prototípico en enunciados cuya forma general marca unívocamente su condición procesual (mediante una función de sujeto marcada temáticamente como objeto afectado o experimentador, propiedades características de los esquemas procesuales) y de procesos no conectados con relación agentiva alguna (a diferencia de los enunciados pasivos-reflexivos). En el marco de un esquema de relaciones semejantes, en el que hechos de valencia tanto específica como genérica señalan la inexistencia de relación agentiva alguna, la cancelación de la valencia de agente, inherente a los verbos realizativos, vendría a ser el resultado de la imposibilidad de precisar el contenido inespecificado de SE, operándose, de este modo, sobre la estructura realizativa prototípica representada en (37), la transclasificación que refleja (47). 


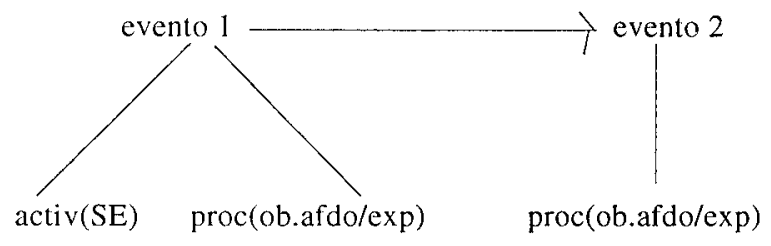

En el marco de una conceptuación de las categorías lingüísticas de orientación prototípica, la utilización parcial del prototipo SE presenta, junto a las posibilidades léxicas que implica su utilización en las estructuras denominadas pasivas e impersonales reflexivas -en las que SE constituye parte del significante complejo de signos de contenido máximamente genérico- otras cuya funcionalidad se sitúa en el ámbito sintáctico-valencial, al permitir la concurrencia de determinadas formas verbales en contextos oracionales que, por su conformación funcional, les estarían vedados. La conjunción 'verbo realizativo $+\mathrm{SE}$ ' constituye, en consecuencia, un procedimiento morfológico regular de ampliación del léxico básico de nuestra lengua, al hacer posible la concurrencia de determinados contenidos léxicos en esquemas oracionales en los que, de otro modo, no podrían ser utilizados.

\section{BIBLIOGRAFÍA:}

Babcock, S. (1970); The Syntax of Spanish Reflexive Verbs. The Parameters of the Middle Voice. La Haya: Mouton.

Báez, V. 1988. Fundamentos críticos de la Gramática de Dependencias. Madrid: Síntesis.

Burzio, L. 1986. Italian Syntax. A Government and Binding Approach. Dordrecht: Reidel. 
Cano Aguilar, R. 1981. Estructuras sintácticas transitivas en el español actual. Madrid: Gredos.

Chomsky, N. 1970. «Remarks on nominalization». R. Jacobs y P. Rosenbaum (eds.): Readings in English Transformational Grammar. Waltam: Ginn.

Demonte, V. 1989. Teoría sintáctica: De las Estructuras a la Rección. Madrid: Síntesis.

Demonte, V. 1991. Detrás de la palabra. Estudios de gramática del español. Madrid, Alianza Universidad.

Dik, S. 1978. Functional Grammar. Amsterdam: North-Holland.

Dowty, D. 1979. Word Meaning and Montague Grammar. Dordrecht: Reidel.

Dowty, D. 1986. «Thematic roles and semantics». Proceedings of the Berkeley

Linguistics Society. 12, págs. 340-354.

Fillmore, Ch. J. 1968. «The case for case». E. Bach y R. Harms (eds.): Universals in Linguistic Theory. N. York: Holt, Rinehart y Winston, págs. 1-88.

Grimshaw, J. 1992. Argument Structure. Cambridge Ma.:The MIT Press.

Gruber, J.S. 1976. Lexical Structures in Syntax and Semantics. Amsterdam:

North-Holland.

Heger, K. 1974. «Deixis personal y persona gramatical». Teoría semántica_II. Madrid: Alcalá, págs. 33-51.

Kenny, A. 1963. Action, Emotion and Will. Londres: Routledge.

Keyser, $\mathbf{J}$ y Roeper, Th. 1984. «On the middle and ergative constructions in English», Linguistic Inquiry 15, págs. 381-346.

Lakoff, G. 1977. «Linguistic Gestalts». Proceedings of the Chicago Linguistics Society. 13.

Lázaro Mora, F. 1983. «Observaciones sobre el «se» medio». Serta Philologica F. Lázaro Carreter. I. Madrid: Cátedra, págs. 301-308.

Martín Zorraquin, María A. 1979. Las construcciones pronominales en español. Madrid: Gredos.

Molina Redondo, J. A. 1974. Usos de se. Madrid: SGEL.

Moreno, J.C. 1984. «La diátesis anticausativa. Ensayo de sintaxis general». RSEL.14,1, págs. 21-42.

Oca, E. 1914. «El pronombre «se» en nominativo». BRAE,1, págs. 573-581.

Reichenkron, G. 1933. Passivum, Medium und Reflexivum in den Romanischen Sprachen. Jena-Lepizig: W. Gronan.

Roca Pons, J. 1970. Introducción a la gramática. Barcelona: Teide.

Tesnière, L. 1976. Éléments de syntaxe structurale. París. Klincksieck.

Vendler, Z. 1967. Linguistic in Philosophy. N. York: Cornell University Press. Vendler, Z. 1984. «Adverbs of Action». Proceedings of the Chicago Linguistics Society; 297-307.

Vera Luján, A. 1990. Las construcciones pronominales pasivas e impersonales en español. Murcia: Secretariado de Publicaciones de la Universidad de Murcia. 
Zubizarreta, María L. 1987. «Niveles de representación léxica: estructura léxico-semántica y estructura léxico-sintáctica», en V. Demotne y María Fernández Lagunilla (eds.): Sintaxis de las lenguas románicas. Madrid: Ed. el arquero; 33-59. 